https://doi.org/10.46344/JBINO.2020.v09i05.19

\title{
IDENTIFYING A POTENTIAL DRUG FROM ACORUS CALAMUS AND DETERMINING THE SPECIFIC BINDING REGION IN THE HUMAN CORONAVIRUS PROTEIN VIA INSILICO DOCKING METHOD.
}

\section{Venkataramanan Swaminathan \& Devindren Subramaniam}

Faculty of Health and Life Sciences, Management and Science University, Seksyen 13, 40100 Shah Alam, Selangor, Malaysia

Department of Diagnostic and Allied Health Science, Faculty of Health and Life Sciences, Management and Science University, 40100, Shah Alam, Selangor, Malaysia.

(Received on Date: $15^{\text {th }}$ June 2020

Date of Acceptance: 31 July 2020

Date of Publish: 1st September 2020)

Email: $\underline{\text { s_venkataramanan@msu.edu.my }}$

\begin{abstract}
An outbreak of 2019 novel coronavirus disease (COVID-19/ 2019-nCoV) has quickly spread nationwide in Wuhan, China. It has been classified as a zoonotic coronavirus similar to coronavirus SARS and coronavirus MERS. Aim: To identify a potential drug and determine the specific binding region in the human coronavirus protein via insilico docking method. Materials and Methods: There is a herb called Acorus Calamus which contains various compounds in it. Hence, one of the most widely known ligand is galangin which is used as a therapeutic target for the treatment of cancer, neurodegeneration, diabetes, cardiovascular effects and also bronchitis diseases. Thus, the ligand was taken from the PubChem database and downloaded in the SDF form. Then, the liability of the ligand was tested through the PreADMET database. On the other hand, human coronavirus protein was downloaded from NCBI VIRUS in FASTA format. Next, by using RPBS WEB PORTAL online server proper docking was done with the chosen receptor and ligand. Results and Discussions: Galangin was recognized as one of the main ligand which can able to bind to a specific region in human coronavirus protein. Conclusion: From this study, galangin was identified as one of the useful ligand to cure COVID-19. This result suggests the potential role of galangin and this ligand to serve as a useful drug in the medical field.
\end{abstract}

Keywords: Nil

No: of Figures: 04

No: of References:10 


\section{INTRODUCTION}

SARS has caused international concern due to its novelty, communicability and rapid spread by jet travel and caused illness in a significant proportion of the medical and nursing staff exposed to it. We do not know where we are on the path of the epidemic. As the epidemic continues, gratitude is due to the hundreds of healthcare workers around the world who come every day to work to assist SARS patients despite certain threats to their health [Holmes, K. V., 2003].

Moreover, the 2019 coronavirus disease (COVID-19) virus, which arose in December 2019, has quickly spread, with cases now confirmed in several countries. The virus had caused 82447 infections and 3310 deaths in mainland China as of 30 th March 2020, meanwhile, 2470 confirmed cases and 34 deaths in Malaysia [Holmes, K. V., 2003].

Much effort has been made in China to find appropriate drugs to fight the virus. On 17 February 2020, the Chinese State Council conducted a news briefing stating that chloroquine phosphate, an old medicine used to treat malaria, had demonstrated marked effectiveness and acceptable protection in the treatment of COVID-19 related pneumonia in multicenter clinical trials in China [Gao, J., 2020].

The documented diseases ranged from mild symptoms to serious disease and death in cases of confirmed coronavirus disease 2019 (COVID-19). Hence, these symptoms will show up 2-14 days after exposure based on the MERS-COV virus incubation period. The first symptom would arise in body temperature up to $380 \mathrm{C}$ and eventually increases until the infected person loses lots of body water content. Next, the person will have a continuous cough which can lead to shortness of breath. The last stage or emergency warning signs for COVID-19 are trouble breathing, persistent pain or pressure in the chest, new confusion or inability to arouse with bluish lips or face, at this stage the person should immediately look for treatment by specialized doctors [Sohrabi, C., 2020].

COVID-19's clinical properties include dry cough, nausea, diarrhea, vomiting, and myalgia. Individuals with several comorbidities are vulnerable to serious infection, and may also have acute kidney injury (AKI) and ARDS characteristics [Sohrabi, C., 2020].

Moreover, there are currently few studies that describe COVID-19's pathophysiological characteristics, and there is significant uncertainty about its spreading mechanism. Current information is primarily derived from related coronavirus, which is transmitted by respiratory fomites from human to human. When a patient becomes symptomatic, respiratory viruses usually are most infectious. There is, however, a growing body of evidence to indicate that human-to-human transmission can occur during the COVID-19 asymptomatic incubation period, estimated to be between 2 and 10 days [Sohrabi, C., 2020]. 


\section{MATERIALS}

In response to the increasing need for a public, virus-specific reference sequence tool, the NCBI Viral Genomes Project was established [Brister, J. R., 2014]. There is a total of six samples taken from the NCBI virus database. Hence, all the samples have been filtered by the host and nucleotide completeness specifications. Thus, one Homo (humans), taxid:9605 (465) sequence and five Chiroptera (bats), taxid:9397 (192) sequences were chosen as the host and for the nucleotide completeness, completed has been chosen. Thus, from this specific filter option, we can get the number of samples. The samples with an accession number of; AAZ67055.1, AAZ67053.1, ABD75315.1, AAZ67039.1, AAP41047.1, AAZ67049.1 has been chosen to proceed further.

\section{METHODS}

\section{NCBI VIRUS DATABASE}

An online survey program designed to support researches who are interested in viral classification and prediction. The database contains all the viral genomes, their corresponding natural vectors, and classification information for the single/multiple-segmented viral reference sequences downloaded from the National Center for Biotechnology Data. This database can be accessed through https://www.ncbi.nlm.nih.gov/la bs/virus/vssi/\#/ website which has played a main role throughout the process. This database was used to extract all the six coronavirus protein sequences. Hence, the coronavirus protein that was selected from the search query was (Severe acute respiratory syndrome-related coronavirus, taxid;694009).

\section{PHYLOGENETIC TREE}

From the six selected protein sequences, we can form a phylogenetic tree from the virus database itself. This phylogenetic tree shows the genetic variations that had to occur between all these protein sequences. This phylogenetic tree is a diagram describing the evolutionary relationships between humans and other organisms. Phylogenetic trees are not conclusive evidence nor theories. In a phylogenetic tree, the pattern of branching illustrates how organisms or other groups developed from a set of similar ancestors.

\section{MODELING SOFTWARE}

Hence, the human coronavirus protein sequence was downloaded from the NCBI VIRUS database in FASTA format and then protein modeling was done. From the online

website

SWISS-MODEL https://swissmodel.expasy.o $\mathrm{rg} / \mathrm{we}$ can create a protein model according to its database algorithm. It is a fully automatic, homology-modeling application for protein structures. Methods of homology modeling consist of the following four steps: (i) template selection; (ii) target template alignment; (iii) model construction; and (iv) evaluation [Schwede, T., 2003]. This platform aims to make protein modeling available for all life.

\section{ADMET}

Analysis of the ADMET (Absorption, Delivery, Metabolism, Excretion \& Toxicity) is the pharmacokinetics of a drug molecule. We currently have a range of online and offline computational tools that help predict and evaluate the ligands 'ADME profiles based on their 
structure and the interaction between the ligands and the receptors [Nisha, C. M., 2016]. ADMET database helps users to identify and calculates the toxicity, ADME, and drug-likeness of the specifically chosen ligands. This test has to be done to ensure that the ligands are not harmful to be used for medical purposes during the clinical test and to ensure that the ligands have the ability to treat certain diseases with no side effects.

\section{DOCKING (RPBS WEB PORTAL)}

Protein-ligand docking is a method for molecular modeling. Protein-ligand docking is intended to predict a ligand's position and orientation when it is bound to a protein receptor or enzyme. Thus, from

this

website https://mobyle.rpbs.univ-paris-did erot.fr/cgi-bin/portal.py\#welcome we can undergo the docking method more simply and easily. Hence, the ligand galangin was downloaded from the PubChem database in SDF 2D format which was used to upload with the same format in the RPBS WEB PORTAL database. On the other hand, the human coronavirus protein was uploaded in PDB format to undergo the docking method.

\section{RESULTS AND DISCUSSION}

Multiple Alignment

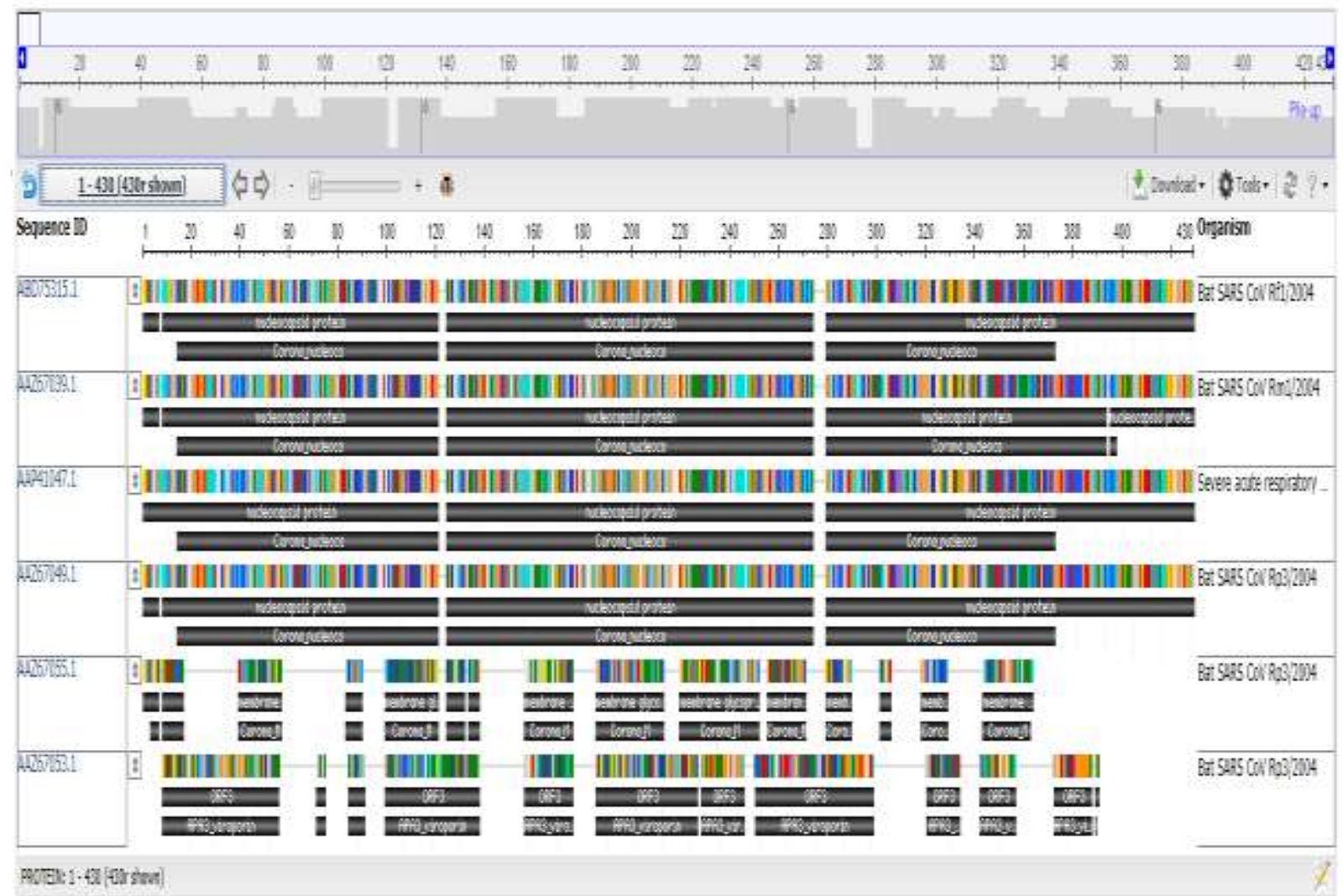

FIGURE 1: Shows the sequence alignment of all the six Severe Acute Respiratory Syndrome protein sequences. 
Series similitude, structural consistency, and length of alignment are noted for each alignment. Homology methods are the most effective and are focused on the identification of significantly extended sequence similarity to a known structure protein or a protein family sequence pattern [Sander, C., 1991]. Hence, from this alignment of all the five Chiroptera bat species and human species, we can see the similarities between coronavirus protein in all the sequences. Thus, we can identify the mismatches, gaps, point mutations that occurred in the sequences

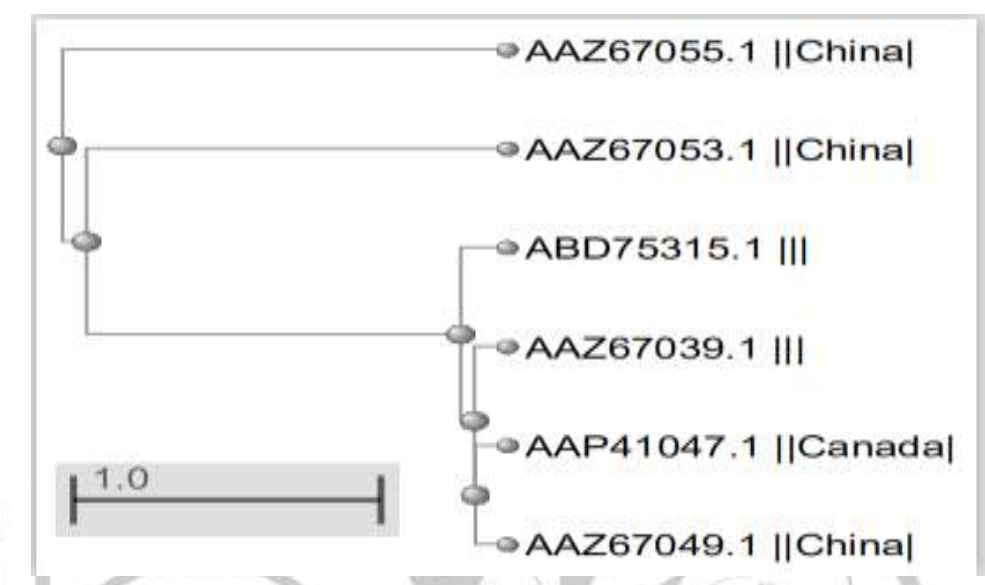

Figure 2: Shows the phylogenetic tree of all the six Severe Acute Respiratory Syndrome protein sequences.

Phylogenetics is important because it enriches our understanding of how genes, genomes, organisms and more generally molecular sequences evolve. Through phylogenetics, we learn not just how the sequences have become the way they are today but also general principles that allow us to predict how they will change in the future [Feng, D.-F., 1990]. Hence, from the above diagram, we can see that the AAZ67005.1 sequence has a separate root since the beginning and no branches or internal nodes formed. Next, AAZ67053.1 has a branch without any internal nodes but only the leaf node is present. Thus, we can say that there is no sequence divergent that occurred in this protein. Meanwhile, the ABD75315.1 sequence has a divergent point at the branch where some point mutation might occur separating and forming three different sequences which are AAZ67039.1, AAP41047.1, AAZ67049.1. 


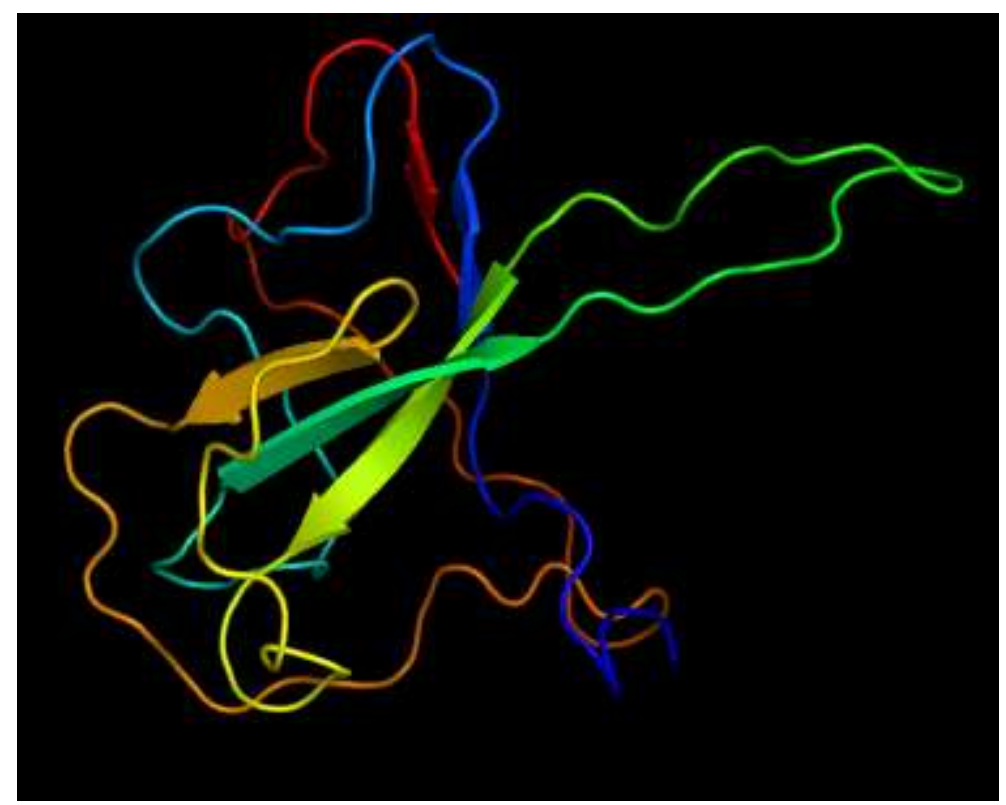

\section{FIGURE 3: Shows human protein modelling using SWISS-MODEL database.}

The SWISS-MODEL server is mainly used in a fully automated mode in which no user input is needed, other than the protein sequence to model. This is the simplest and most user-friendly way to get a protein pattern. Proteins of different origins may have very similar sequences, and it is widely agreed that high sequence similarity is expressed by distinct similarity in structure[Guex, N., 1997]. However, in this coronavirus protein modeling, we can determine the structure and loops present in the model above. This prediction will allow us to proceed with the next step which is docking.
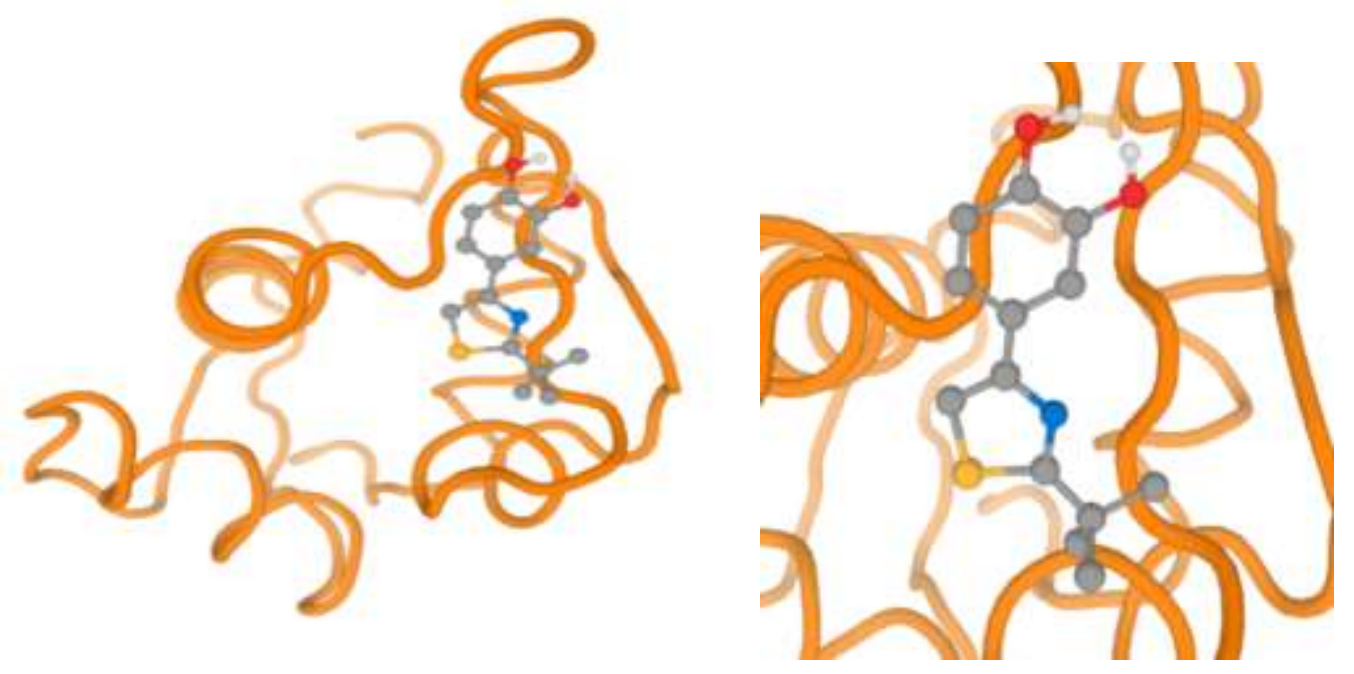

FIGURE 4: Shows the protein-ligand binding viewed from the RPBS WEB PORTAL 
RPBS provides many other structure-focused services, including secondary structure classification, solvent accessibility, and side-chain modeling. By creating a Phyton API, RPBS contributed to the bionomics project and began providing such choices in the form of a web service [Guex, N., 1997]. Computational identification of small molecule binding sites has many significant applications in the field of biomedicine. The detection of cavities for structural drug discovery or functional annotation of structures is notable interests[Schmidtke, P., 2010]. Thus, from figure 4 we can see that the ligand galangin from the herb Acorus calamus can bind into the specific region of the Severe Acute Respiratory Syndrome human protein pocket.

\section{CONCLUSION}

In a nutshell, from this research, we can identify a potential drug which might be a useful part for medical purpose in curing the coronavirus Severe Acute Respiratory Syndrome. Hence, we have identified a useful ligand named galangin from the herb Acorus calamus that could bind to the specific pocket in the human coronavirus protein.

\section{REFERENCES}

Brister, J. R., Ako-Adjei, D., Bao, Y., \& Blinkova, O. (2014). NCBI Viral Genomes Resource. Nucleic Acids Research, 43(D1). doi: 10.1093/nar/gku1207

Feng, D.-F., \& Doolittle, R. F. (1990). [23] Progressive alignment and phylogenetic tree construction of protein sequences. Methods in Enzymology,
10.1016/0076-6879(90)83025-5

Gao, J., Tian, Z., \& Yang, X. (2020). Breakthrough: Chloroquine phosphate has shown apparent efficacy in treatment of COVID-19 associated pneumonia in clinical studies. Bioscience Trends, 14(1), 72-73. doi: 10.5582/bst.2020.01047

Guex, N., \& Peitsch, M. C. (1997). SWISS-MODEL and the Swiss-Pdb Viewer: An environment for comparative protein modeling. Electrophoresis, 18(15),

2714-2723. doi: 10.1002/elps.1150181505

Holmes, K. V. (2003). SARS-Associated Coronavirus. New England Journal of Medicine, 348(20), 1948-1951. doi: $10.1056 /$ nejmp030078

Nisha, C. M., Kumar, A., Vimal, A., Bai, B. M., Pal, D., \& Kumar, A. (2016). Docking and ADMET prediction of few GSK-3 inhibitors

divulges

6-bromoindirubin-3-oxime as a potential inhibitor. Journal of Molecular Graphics and Modelling, 65, 100-107. doi: 10.1016/j.jmgm.2016.03.001

Sander, C., \& Schneider, R. (1991). Database of homology-derived protein structures and the structural meaning of sequence alignment. Proteins: Structure, Function, and Genetics, 9(1), 56-68. doi: 10.1002/prot.340090107

Schmidtke, P., Guilloux, V. L., Maupetit, J., \& Tuffery, P. (2010). fpocket: online tools for protein ensemble pocket detection and tracking. Nucleic Acids Research, 38(Web Server). doi: 10.1093/nar/gkq383

Schwede, T. (2003). SWISS-MODEL: an automated protein homology-modeling 
server. Nucleic Acids Research, 31 (13), 3381-3385. doi: 10.1093/nar/gkg520

Sohrabi, C., Alsafi, Z., Oneill, N., Khan, M., Kerwan, A., Al-Jabir, A., ... Agha, R. (2020). World Health Organization declares global emergency: A review of the 2019 novel coronavirus (COVID-19). International Journal of Surgery, 76, 71-76. doi: 10.1016/j.ijsu.2020.02.034 